\title{
Influence of Electron Correlation Effects on the Solvation of $\mathrm{Cu}^{2+}$ Supplementary Material
}

\author{
Christian F. Schwenk and Bernd M. Rode ${ }^{*}$ \\ Institute of General, Inorganic and Theoretical Chemistry \\ University of Innsbruck, Innrain 52a, A-6020 Innsbruck, Austria \\ E-Mail: bernd.m.rode@uibk.ac.at \\ Tel.: +43-512-507-5160 \\ Fax: +43-512-507-2714
}

31st August 2004

${ }^{*}$ Corresponding author

$¥$ This work was supported by the Austrian Science Foundation (FWF); project P16221-NO8. 
Table 1: Optimized parameters for the $\mathrm{Cu}-\mathrm{O}$ and $\mathrm{Cu}-\mathrm{H}$ two-body functions calculated at $\mathrm{HF}$ level (energies in $\mathrm{kcal} \mathrm{mol}^{-1}$, distances in $\AA$ ).

\begin{tabular}{cccccc}
\hline & $A$ & $B$ & $C$ & $D$ & $E$ \\
\hline $\mathrm{Cu}-\mathrm{O}$ & -7445.3173 & 18631.6041 & -10033.7184 & -14333.9540 & 3.6850725 \\
$\mathrm{Cu}-\mathrm{H}$ & 2646.0777 & -29355.9952 & 24575.6334 & 122857.1021 & 3.4967237 \\
\hline
\end{tabular}

\section{Details of Calculations}

\subsection{Evaluation of the two-body potential function}

The two-body energy hypersurface $\left(\mathrm{E}_{2 \mathrm{bd}}=\mathrm{E}_{\mathrm{Cu}-\mathrm{H}_{2} \mathrm{O}}-\mathrm{E}_{\mathrm{Cu}}-\mathrm{E}_{\mathrm{H}_{2} \mathrm{O}}\right)$ was evaluated to construct the potential for the $\mathrm{Cu}^{\mathrm{II}}-\mathrm{H}_{2} \mathrm{O}$. Ab initio molecular orbital calculations were carried out at the unrestricted Hartree-Fock (UHF) level using the TURBOMOLE program. ${ }^{1-4}$ The LANL2DZ effective core potential (ECP) basis set for $\mathrm{Cu}^{\mathrm{II} 5}$ and the DZP basis set for $\mathrm{H}_{2} \mathrm{O}^{6}$ were employed, providing a proven ${ }^{7-9}$ satisfactory compromise between accuracy and efficiency.

The energy hypersurface was then fitted to a chosen functional form by a least square error minimization using the Levenberg-Marquardt algorithm. The best reproducibility was obtained using a function composed of three $r^{-m}$ and one exponential term for the non-Coulombic interaction in addition to the Coulombic interaction, as expressed by Equation 1,

$$
\begin{aligned}
E_{2 \mathrm{bd}}= & \frac{q_{O} q_{C u^{2+}}}{r}+\frac{A_{O}}{r^{4}}+\frac{B_{O}}{r^{5}}+\frac{C_{O}}{r^{6}}+D_{O} \exp \left(-E_{O} r\right)+ \\
& \sum_{i=1}^{2} \frac{q_{H} q_{C u^{2+}}}{r_{i}}+\frac{A_{H}}{r_{i}{ }^{4}}+\frac{B_{H}}{r_{i}{ }^{6}}+\frac{C_{H}}{r_{i}{ }^{7}}+D_{H} \exp \left(-E_{H} r_{i}\right)
\end{aligned}
$$

where $A, B, C, D$ and $E$ are the final fitting parameters summarized in Table 1. $q_{O}$ are the partial charges of oxygen and hydrogen, set to -0.65966 and $0.32983^{10}$ according to the BJH-CF2 ${ }^{11,12}$ water model, and $q_{\mathrm{Cu}^{2+}}$ is the charge on the copper(II) ion, set to +2.0 . The experimental gas phase geometry of $\mathrm{H}_{2} \mathrm{O}$ was fixed with the $\mathrm{O}-\mathrm{H}$ distance of $0.9601 \AA$ and the $\mathrm{H}-\mathrm{O}-\mathrm{H}$ angle of 
$104.47^{\circ} \cdot{ }^{13} E_{2 \mathrm{bd}}$ values near the global minimum were emphasized during the least-squares procedure by assigning an appropriate weighting factor and $E_{2 \mathrm{bd}}$ values above $30 \mathrm{kcal} \mathrm{mol}^{-1}$ were excluded from the fitting procedure. The average absolute residual of the fit was $1.8 \mathrm{kcal} \mathrm{mol}^{-1}$ and the global energy minimum of the fitted function was found to be $-91.9 \mathrm{kcal} \mathrm{mol}^{-1}$.

\subsection{Evaluation of the three-body potential function}

The three-body correction term was constructed according to Equation 2,

$$
E_{3 \mathrm{bd}}^{\mathrm{corr}}=E_{\mathrm{CuW}^{i} \mathrm{~W}^{j}}-\left(E_{\mathrm{Cu}}+2 E_{\mathrm{W}}\right)-\left(E_{\mathrm{CuW}^{i}}+E_{\mathrm{CuW}^{j}}\right)-E_{\mathrm{W}^{i} \mathrm{~W}^{j}}
$$

where $E_{\mathrm{CuW}^{i} \mathrm{~W}^{j}}$ is the $\mathrm{SCF}$ energy for $\left[\mathrm{Cu}\left(\mathrm{H}_{2} \mathrm{O}\right)_{2}\right]^{2+}, E_{\mathrm{CuW}^{i}}$ and $E_{\mathrm{CuW}^{j}}$ are the two-body energies calculated using Equation 1, and $E_{\mathrm{W}^{i} \mathrm{~W}^{j}}$ is the interaction energy between water molecules.

To construct the three-body potential we evaluated the energy hypersurface for $\mathrm{H}_{2} \mathrm{O}-\mathrm{Cu}^{\mathrm{II}}-$ $\mathrm{H}_{2} \mathrm{O}$ by ab initio molecular orbital calculations at UHF level. For the geometries employed in this work almost all $E_{3 \mathrm{bd}}^{\text {corr }}$ values were found to be positive (repulsive) decreasing with growing ion-oxygen distances and approaching $0 \mathrm{kcal} \mathrm{mol}^{-1}$ at a distance of $6.0 \AA$. The resulting energies were fitted to the analytical formula:

$$
\begin{aligned}
& E_{3 \mathrm{bd}}^{\text {corr }}=A \exp \left(-B r_{12}\right) \exp \left(-B r_{13}\right) \exp \left(-C r_{23}\right) \\
& {\left[\left(r_{\text {limit }}-r_{12}\right)^{2}\left(r_{q q \text { limit }}-r_{13}\right)^{2}\right]}
\end{aligned}
$$

with the fitting parameters $A, B$ and $C$. The term in square brackets ensures that the energy for all distances above $r_{\text {limit }}$, i.e. $6.0 \AA$, vanishes. The other variables are the $\mathrm{O}-\mathrm{O}$ distance, $r_{23}$, and the distances between the copper ion and the first $\left(r_{12}\right)$ and the second $\left(r_{13}\right)$ oxygen. A total of 10000 configurations were generated and the analytical function of Equation 3 was fitted to the $E_{3 \text { bd }}$ values by a least-squares optimization achieving an average absolute residual of the fit of 
Table 2: Optimized parameters $A, B$ and $C$ of the three-body correction function, Equation 3 (energies in $\mathrm{kcal} \mathrm{mol}^{-1}$, distances in $\AA$ ).

\begin{tabular}{ccc}
\hline$A$ & $B$ & $C$ \\
\hline 0.0441710 & 0.0576117 & 0.0315034 \\
\hline
\end{tabular}

$2.0 \mathrm{kcal} \mathrm{mol}^{-1}$. The optimized parameters for the three-body correction function are summarized in Table 2.

\subsection{Simulation protocol}

The simulation protocol for all molecular dynamics (MD) simulations was the same as in previous works. ${ }^{9,14,15}$ The simulations were carried out in a canonical $N V T$ ensemble consisting of one $\mathrm{Cu}^{\mathrm{II}}$ ion and 499 water molecules at $298 \mathrm{~K}$ using a cubic box of side length $24.6 \AA$. The temperature was kept constant by the Berendsen algorithm ${ }^{16}$ with a relaxation time of $0.1 \mathrm{ps}$. The density of the system was assumed to be $0.997 \mathrm{~g} \mathrm{~cm}^{-3}$, which is the density of pure water at the simulation temperature. Periodic boundary conditions were applied to the simulation box and the reaction field procedure ${ }^{17}$ was employed in all simulations to correct the cutoff of long-range electrostatic interactions. For water-water interactions the flexible BJH-CF2 water model $^{10,11}$ was used, consisting of intermolecular and intramolecular potentials. The cutoff for non-Coulombic interactions between $\mathrm{H}$ atoms and between $\mathrm{O}$ and $\mathrm{H}$ was set to $3.0 \AA$ and $5.0 \AA$, respectively, and all other pair interactions were cut off at $12.0 \AA$. The pair potentials and forces were calculated within the spherical cutoff limit, and to avoid discontinuity of the potential function at the cutoff distance the shifted-force potential was used. ${ }^{18}$ The Newtonian equations of motion were treated by a general predictor-corrector algorithm and a time step of 0.2 fs was chosen, since the BJH-CF2 water model allows explicit hydrogen movements.

In order to construct the initial configuration, the $\mathrm{O}$ atoms of $\mathrm{H}_{2} \mathrm{O}$ were placed in the simu- 
lation box according to the face-centered cubic lattice structure and the $\mathrm{H}$ atoms were arranged with random orientations of $\mathrm{H}_{2} \mathrm{O}$ molecules. Equilibration was performed for 300.000 steps after which the system was confirmed to be energetically equilibrated. A further 500.000 steps were sampled to evaluate structural properties before, and a further 500.000 steps after the inclusion of three-body corrections.

\section{$1.4 \mathrm{QM} / \mathrm{MM}-\mathrm{MD}$ simulation}

The QM/MM MD simulation was performed describing the complete first shell up to $3.2 \AA$ at the UHF level using the same basis sets as employed in the construction of pair and three-body potentials. The water molecules in the outside region were treated by the MM potentials (twoplus three-body). In QM/MM MD simulations the force can be written as

$$
F_{\mathrm{tot}}=F_{\mathrm{MM}}^{\mathrm{sys}}+\left(F_{\mathrm{QM}}^{\mathrm{QM}}-F_{\mathrm{QM}}^{\mathrm{MM}}\right) * S(r)
$$

where $F_{\text {tot }}$ is the total force of the system, $F_{M M}^{\text {sys }}$ is the $M M$ force of the total system, $F_{\mathrm{QM}}^{\mathrm{QM}}$ is the $\mathrm{QM}$ force in the $\mathrm{QM}$ region, and $\mathrm{F}_{\mathrm{QM}}^{\mathrm{MM}}$ is the $\mathrm{MM}$ force in the $\mathrm{QM}$ region. $\mathrm{F}_{\mathrm{QM}}^{\mathrm{MM}}$ accounts for the coupling between QM and MM region. The smoothing function S ensures a continuous transition from $\mathrm{QM}$ to $\mathrm{MM}$ region. The $\mathrm{QM} / \mathrm{MM} \mathrm{MD}$ program used in this work allows water molecules to leave and enter the QM region as needed to be able to fully describe the dynamics of the system. The radius of the QM region was estimated according to the radial distribution function (RDF) obtained by the classical MD simulation including three-body corrections.

The QM/MM MD simulation was started from the equilibrated configuration of the previous two QM/MM MD simualtion including an additional three body potential in the classical region which was performed for more than 30 ps. Another 5000 steps of equilibration were performed and the subsequent MP2/MM simulation was continued for further 25000 time steps to collect 
configurations every 5th step. The QM/MM MD simulation was performed on 5 dual processors (Athlon $2.4 \mathrm{GHz}$ ) consuming 150 days of CPU time. All quantum-mechanical calculations were performed by the parallelized version of the TURBOMOLE program package. ${ }^{1-4}$

\subsection{Ion-Oxygen Frequencies and Mean Residence Times}

Using the approximative normal coordinate analysis, ${ }^{19,20}$ velocity autocorrelation functions (VACFs) and their Fourier transformed power spectra provide basic informations about the dynamics of hydrated ions. The frequencies of water molecules in the quantum mechanically treated first (and second) hydration shell were multiplied by a standard HF scaling factor of $0.89(\mathrm{HF}){ }^{15,21,22}$

In a previous investigation ${ }^{23}$ we have compared two different evaluation methods for mean ligand residence times, namely the "direct" method, accounting for all actually incoming and outgoing ligands, and the procedure suggested by Impey et al., ${ }^{24}$ based on a "survival function", and we have shown the "direct" method to be more precise and thus advantageous. The most appropriate time span to record a ligand displacement from its original coordination sphere as an exchange process is $0.5 \mathrm{ps}$ (this time also corresponds to the average life time of a hydrogen bond in the solvent ${ }^{25}$ ).

\section{References}

(1) Ahlrichs, R.; Bär, M.; Häser, M.; Horn, H.; Kölmel, C. Chem. Phys. Lett. 1989, 162(3), $165-169$.

(2) Brode, S.; Horn, H.; Ehrig, M.; Moldrup, D.; Rice, J. E.; Ahlrichs, R. J. Comput. Chem. 1993, 14(10), 1142-1148. 
(3) Ahlrichs, R.; von Arnim, M. in Methods and Techniques in Computational Chemistry: METECC-95, Clementi, E.; Corongiu, G., Eds., chapter 13, pp 509-554. STEF, Cagliari 1995.

(4) von Arnim, M.; Ahlrichs, R. J. Comput. Chem. 1998, 19(15), 1746-1757.

(5) Wadt, W. R.; Hay, P. J. J. Chem. Phys. 1985, 82, 284-298.

(6) Dunning, T. H. Jr. J. Chem. Phys. 1970, 53(7), 2823-2833.

(7) Inada, Y.; Mohammed, A. M.; Loeffler, H. H.; Rode, B. M. J. Phys. Chem. A 2002, 106(29), 6783-6791.

(8) Loeffler, H. H.; Rode, B. M. J. Chem. Phys. 2002, 117(1), 110-117.

(9) Schwenk, C. F.; Loeffler, H. H.; Rode, B. M. J. Chem. Phys. 2001, 115(23), 10808-10813.

(10) Stillinger, F. H.; Rahman, A. J. Chem. Phys. 1978, 68(2), 666-670.

(11) Bopp, P.; Janscó, G.; Heinzinger, K. Chem. Phys. Lett. 1983, 98(2), 129-133.

(12) Lemberg, H. L.; Stillinger, F. H. J. Chem. Phys. 1975, 62(5), 1677-1690.

(13) Kuchitsu, K.; Morino, Y. Bull.Chem.Soc.Jpn 1965, 38, 814.

(14) Tongraar, A.; Liedl, K. R.; Rode, B. M. J. Phys. Chem. A 1998, 102(50), 10340-10347.

(15) Schwenk, C. F.; Loeffler, H. H.; Rode, B. M. J. Am. Chem. Soc. 2003, 125, 1618-1624.

(16) Berendsen, H. J. C.; Postma, J. P. M.; van Gunsteren, W. F.; DiNola, A.; Haak, J. R. J. Phys. Chem. 1984, 81, 3684-3690.

(17) Adams, D. J.; Adams, E. M.; Hills, G. J. Mol. Phys. 1979, 38(2), 387-400. 
(18) Allen, M. P.; Tildesley, D. J. Computer Simulation of Liquids. Oxford Science Publications 1990.

(19) Bopp, P. Chem. Phys. 1986, 106(205-212).

(20) Spohr, E.; Pálinkás, G.; Heinzinger, K.; Bopp, P.; Probst, M. M. J. Phys. Chem. 1988, 92(23), 6754-6761.

(21) DeFrees, D. J.; McLean, A. D. J. Chem. Phys. 1985, 82(1), 333-341.

(22) Scott, A. P.; Radom, L. J. Phys. Chem. 1996, 100, 16502-16513.

(23) Hofer, T. S.; Tran, H. T.; Schwenk, C. F.; Rode, B. M. J. Comput. Chem. 2004, 25, 211-214.

(24) Impey, R. W.; Madden, P. A.; McDonald, I. R. J. Phys. Chem. 1983, 87(25), 5071-5083.

(25) Lock, A. J.; Woutersen, S.; Bakker, H. J. Femtochemistry and Femtobiology. World Scientific 2001. 